\title{
NUMERICAL STUDY OF THE SECONDARY CIRCULATION FLOW IN A VALLEY*
}

\author{
Marcelino Ferro - M.Sc. student \\ Departamento de Fisica - Centro de Ciências Naturais e Exatas \\ UFSM, Santa Maria, RS
}

Marcelo Moraes - M.Sc. student

Centro de Pesq.em Sens. Remoto e Meteorologia

UFRGS, Porto Alegre, RS

Osvaldo L. L. Moraes - Advisor Professor

Departamento de Física - Centro de Ciências Naturais e Exatas

UFSM, Santa Maria, RS

\begin{abstract}
In this paper it was numerically simulated an air stream perpendicularly incident upon a valley in order to determine the influence of the Froude and the Rossby numbers in the pattern of the flow. The main of this study is to search under what range of these parameters the air stream will be channeled in the valley. Theoretical considerations shows that the channeling occur if the values of these numbers are limited in one region in the space defined by these parameters.
\end{abstract}

RESUMO

Neste artigo simula-se numericamente um escoamento atmosférico que incide perpendicularmente sobre um vale. $\mathrm{O}$ objetivo do presente trabalho é determinar a influência dos números de Froude e de Rossby sobre o padrão ondulatório resultante bem como o intervalo destes parâmetros que possibilitam a canalização do escoamento. Considerações teoricas permitem inferir . que tal canalização restringe-se a uma certa região do espaço de fase criado por estes parâmetros.

*Supported in part by Conselho Nacional de Desenvolvimento Cientifico e Tecnologico (CNPq), Fundação de Amparo a Pesquisa do Estado do Rio Grande do Sul (FAPERGS) and Comissão de Aperfeiçoamento do Pessoal de Ensino Superior ( CAPES). 


\section{INTRODUCTION}

The character of air flow over valleys and mountains depends on the measure of the stratification and on the horizontal and vertical scales of the orographic features, or more precisely on the Froude number and on the Rossby number defined respectively as $U / N h$ and $U / f L$, where $U$ is the speed of the oncoming flow, $N$ is the Brunt-Vaisala frequency, $f$ is the Coriolis parameter and $h$ and $L$ are the height and width of the orography.

For flow over small-scale orographic features at large Rossby number (of order ten or more), the earth's rotation has little effect, and the theory of the resulting wind field pattern can be simplified by ignoring the rotation altogether. At the opposite end of the spectrum, flow over extensive orographic areas at small Rossby numbers, simplification is achieved by utilizing the quasigeostrophic character of the flow. In the middle part of the spectrum the flow in this range of the Rossby number is strongly affected by the earth's rotation, but is not quasi-geostrophic. Therefore neither of the simplifications mentioned above are applicable. For $R_{o} \propto 1$ studies suggest that both the geostrophic and ageostrophic signals should be evident in the flow response. Stratified flow past an obstacle for $F_{r} \propto 0.1-0.5$ is also not covered by existing theories (Smolarkiewikz and Rotunno 1988). Linear gravity-wave theories are formally valid when $F_{r} \gg>1$ while potential flow-type theory is valid when $F_{r} \ll<1$.

Recourse to simple theoretical models to provide further understanding of these flow phenomena in the $R_{o} x F_{r}$ space of parameters is hampered by the inevitable non-linearity in some part of this space. Thus numerical nonlinear models is the way to gain access to the dynamics of the flow and to simulated linear and nonlinear aspects of the flow

In the present numerical work we study specifically the relationship between the flow along the valley axis and the Froude and Rossby numbers. In other words we examine for what range of these parameters we will obtain the channeling effect when the geostrophic wind is perpendicular to the flow. In this investigation we utilize a system of equations governing hydrostatic flow of an inviscid, incompressible, rotating, density stratified fluid in the isosteric coordinates.

Much of the early ideas and conceptual models of valley winds was rooted in the Defant's scheme (1951) which is a concise overview of classical field work in the Alps. His scheme depicting the sequence of upslope and dowslope winds and its importance in driven the along-valley wind. He indicated that this flow along the valley axis were a nonlinear, three-dimensional phenomenon depended upon the three-dimensionality of the valley and intimately related to the side 
wall slope flows and radiative and turbulent exchange processes. Such complexity precluded the application of the analytic mathematical tools available at the time. However in the past two decades considerable progress has been made concerning numerical models partly due to the development of computer capacity and numerical modeling techniques which allow to simulate three-dimensional motions in a much more realistic way. The work of Egger (1981) did examine flow along the valley axis and it address the descriptive ideas of Defant concerning the development of along-valley flow. McNider and Pielke (1984) utilized a hydrostatic, primitive equation model to examine specifically the development of the secondary along-valley axis flow in well defined valleys.

More recently Adrian and Fiedler (1991) used the non-hydrostatic Karlruhe Atmospheric Mesoscale Model to simulate unstationary wind and temperature fields over complex terrain and particularly the surface wind field in the Rhine valley. On the other hand the successful theoretical explanation of the channeling is that Wippermann (1984) which determines the wind direction in the valley depending on the direction of the geostrophic wind. Dergeiner and Dreiseitl (1987) also explain up- and down valley winds as a result of a pressure contrast between plain and valley. This pressure contrast arises from a horizontal temperature gradient which reverses sign twice daily.

In this present work we are not so much interested in the relationship between the slope flow and the axial flow. The main point which is investigated is the influence of the buoyancy and the Coriolis forces in driving the along-valley axis wind. The importance of these forces arises in two natural scales: first, a length scale, the distance of downwind drift during a buoyancy oscillation and second, a time scale, the transition time of an air parcel to cross the valley $(=L / U)$. The first one, when compared with the height of the valley, gives an idea of how much one particle will be deflected vertically while it cross the valley. This comparison is expressed by the Froude Number. The second one, when compared with the Coriolis parameter, gives an idea of the relative importance of fluid accelerations relative to those associated with the Earth's rotation. This comparison is expressed by the Rossby number.

In all of our numerical experiments we consider situations among four well-defined limits. With large Froude and Rossby numbers the pressure field is nearly hydrostatic and the Coriolis Force can be neglected. With small Froude and Rossby numbers the flow is closely analogous to the irrotational (i.e. potential) and the assumption of geostrophic balance is valid. We also study situations with large Rossby and small Froude numbers and vice versa. 


\section{THE MODEL}

The problem under consideration is to determine, for a straight and perpendicular to the valley flow, the development of the along-valley axis flow caused by the prescribed corrugations of the underlying ground surface and the stratification of the oncoming flow.

The starting point of our analysis is the set of equations governing hydrostatic flow of an inviscid, incompressible, density stratified fluid on the rotating earth. In an isosteric coordinates system these equations take the form

$$
\begin{aligned}
\frac{\partial u}{\partial t}+u \cdot\left(\frac{\partial u}{\partial x}\right)_{\alpha}+v \cdot\left(\frac{\partial u}{\partial y}\right)_{\alpha}-f \cdot v+\left(\frac{\partial M}{\partial x}\right)_{\alpha} & =0 \\
\frac{\partial \mathrm{v}}{\partial \mathrm{t}}+u \cdot\left(\frac{\partial v}{\partial x}\right)_{\alpha}+v \cdot\left(\frac{\partial v}{\partial y}\right)_{\alpha}+f \cdot u+\left(\frac{\partial M}{\partial y}\right)_{\alpha} & =0 \\
\frac{\partial^{2} p}{\partial t \partial \alpha}+\frac{\partial}{\partial x}\left(u \cdot \frac{\partial p}{\partial \alpha}\right) \frac{\partial}{\partial y}\left(v \cdot \frac{\partial p}{\partial \alpha}\right) & =0 \\
\frac{\partial \mathrm{M}}{\partial \alpha} & =p
\end{aligned}
$$

where $\alpha=\rho^{-1}$ is the specific-volume coordinate of the model, $\mathrm{p}$ is the pressure, $M=\alpha \cdot p+g . h$ is the Montgomery potential with $h$ and $g$ denoting the actual height of a density surface and the acceleration of gravity, respectively. The subscript $\alpha$ on the derivatives denote differentiation at constant $\alpha$. In the present study $u$ and $v$ are the velocities in $x$ and $y$ directions respectively. We assume that far upstream of the valley the flow consists of a uniform zonal current of velocity $U$ with uniform stratification given by constant Brunt-Vaisala frequency $N$. The valley extended in the $y$-direction and is defined by

$$
h(x)=H\left(1-\left(1+(x / L)^{2}\right)^{12}\right)
$$

The objective of the calculation is to determine steady state patterns in relation to the wind profiles of the air current. This is done by computing the evolution of the motion in time, starting from a chosen initial state, until no longer changes be noticeably. The underlying assumption is that the final steady state does not depend on the initial condition, but only on the boundary conditions. The initial condition is thus immaterial, provided that it does not lead to wind field breaking during the transient stage, which might cause the numerical integration to break down.

An understanding of the physical basis of the phenomenon of the channeling is of utmost importance. Several key questions emerge: under what range of atmospheric stability and for what range of height and width of the valley does the channeling occur? What role does the Coriolis force play in this case? 
Smith (1979) indicates that non linearity is coupled with the phenomena of wave steeping and breaking, and additionally with blocking. Blocked or stagnant air masses below the crest height of a valley may occur, if the Froude-number drops below a certain critical value. We investigate when the stagnation occur, i.e. under what limit of $F_{r}$ we have $u=0$, where $u$ is the transversal velocity to the valley. In this case the Coriolis force begins to play the outmost important factor to the channeling of the flow.

Fitzjarrald and Lala (1989) proposes when there is a significant along-valley pressure gradient (strong cross-valley geostrophic wind component), the effect of the valley sidewalls is to produce a small cross-valley pressure gradient that opposes the Coriolis force. The Coriolis acceleration then might plausibly be balanced by cross-valley pressure gradients induced by a tilt in the thickness of a cooler, denser layer near the surface.

Intuitively, we would expect low-level dense air to be blocked by a sufficiently high valley, provided the air is not moving too swiftly. In this case, inside the valley, the kinetic energy must be only due to $v$ velocity, where $v$ is the parallel velocity to the axis valley.

\section{NUMERICAL ASPECTS}

The numerical model used in this study was developed by Smolarkiewicz (1991) (for further details see Smolarkiewicz and Clark 1986). The model is a finite-difference approximation and it is an extension of schemes refereed in meteorological literature as Crowley-type schemes. The extension here refereed is in the sense that the scheme included arbitrary forcing and/or source terms.

In the horizontal all variables are defined at the same grid-point positions; in the $\alpha$ direction the velocity field and Montgomery potential are staggered with respect to the pressure. The temporal discretization is documented in Smolarkiewicz (1991) and uses the nonlinear MPDATA scheme for the advection terms involved in the equations. This MPDATA scheme compensates the forcing error term which is a consequence of discarding them in the derivation of ${ }^{*}$ the Crowley-type schemes. Another property of the MPDATA is that bounds the total energy which is necessary for the nonlinear stability of the system.

The spatial staggering is achieved by averaging the velocity variable between the two adjacent grid points, whereas the temporal staggering employs the extrapolation of the velocities from $n-1$ and $n$ temporal levels. In order to prevent spurious accelerations due to pressure forces 
from zero-thickness layers the horizontal derivative of the Montgomery potential is approximated with the second-order-accurate pressure-thickness-weighted average of the one-sided derivatives.

The time-step advancement of the discrete equations proceeds in three stages. First, the advective Courant numbers, and the transported variables, are defined using primary variables $u, v$ and $p$ at appropriate temporal and spatial levels; and the advection of the variables is solved using MPDATA algorithm. Second, the new values of pressure are recovered from the updated pressure thickness, and the hydrostatic relationship provides new values of the Montgomery potential. Third, having available the new values of the pressure thickness and Montgomery potentials, the forcing terms in the momentum equation are incorporated using the arithmetic averages from the old and new time levels. Finally, the updated velocity field is recovered from the momentum variable.

In order to simulate an infinite extend of the fluid a gravity-wave absorber is employed in the upper portion of the model; and the symmetric boundary conditions are incorporated at the lateral boundaries. The computational domain cover 25 points in both horizontal directions with igual grid spacing. In the vertical direction we use 13 points with igual grid spacing.

\section{RESULT AND DISCUSSION}

We wish to focus on the way the pattern of the flow depends on $R_{o}$ and $F_{r}$, particularly the pattern of the flow inside the valley. For this analyze a serie of numerical experiments was simulated. These experiments was carried for a large variety of values of $R_{o}$ and $F_{r}$.

The fundamental parameters that define these numbers enters through the physical characteristics of the valley (its height $h$ and its widht $L$ ) as well its geographic position $(f)$, and through the initial state of the atmosphere, i.e. through the Brunt-Vaisala frequency $(N)$ and the far upstream flow speed $(U)$. Thus the numerical integration of equations (1)-(4) were carried until the wave fields were nearly stationary throughout the region of integration.

The results of the numerical integration, for one particular experiment, are shown in figures (1)-(3). This experiment run with the following parameters: $U=8 \mathrm{~m} / \mathrm{s}, N=0.04 \mathrm{~s}^{-1}$, $h=600 \mathrm{~m}, L=30 \mathrm{Km}$, and $f=1.263 \times 10^{-4} \mathrm{~s}^{-1}$ that corresponds a valley located at 60 degrees of latitude north. With these parameters the corespondents Froude and Rossby numbers are equal to 0.33 and 2.11 respectively.

The time step used was $\Delta \mathrm{T}=10 \mathrm{~s}$ and the horizontal grid spacing was $\Delta \mathrm{X}=\Delta \mathrm{Y}=5$ $\mathrm{Km}$. After 540 time steps of integration the results do not change in significative manner, indicating 
that the flow was stationary to a good approximation

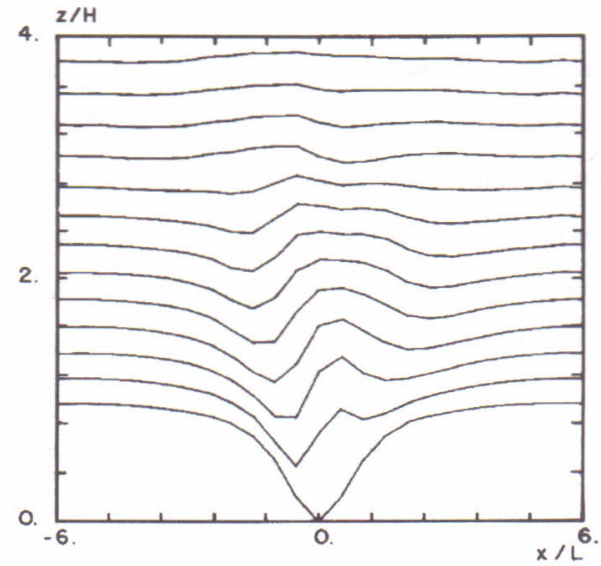

Figure 1. Sketch of the vertical structure of the $\alpha$ surfaces at the center plane for $F_{r}=0.33$ anc $R_{o}=2.11$ The steady state was obtained after 540 steps of integration .

In figure 1 we display the structure of the $\alpha$ surfaces in a vertical cross section through the center plane. This figure shows the propagating-wave pattern with an intrinsic frequency less than the Brunt-Vaisala frequency. In this case internal gravity waves are possible. These waves propagate vertically and the disturbance decay upward. The phase lines tild forward into the mean wind and, as poited by Smith, is connected with the propagation of energy vertically away from the topography that produces the wave.

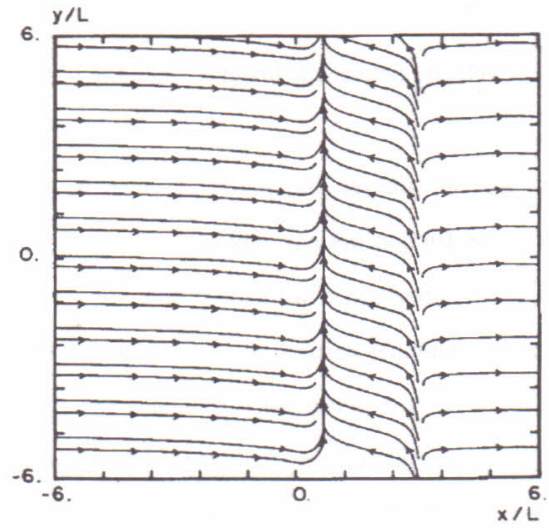

Figure 2. Steady state streamlines at the lower surface for $F_{r}=0.33$ and $R_{o}=2.11$ 
In figure 2 we display the streamlines at the lower surface. In this figure we observe the channelling effect. As a consequence of equations 1-4 the wind follows the $\alpha$ surface, i.e. there are no vertical interactions among the grid points; the wind penetrates the valley and for some physical reason it is accelerates in the axis direction. The strongest $u$-component occurs before the valley center line. Then it decreases and near the valley center line it reaches the zero value. After the center valley its value is negative. In other words the $u$ velocity increase before the axis of the valley, decrease (reaching negative values) and increase again. The increase in the wind speed in the left side of the valley and its decreasing in the right side is an especially interesting facet of the model. We observe $u=0$ in two lines. The first one is located near the center valley axis and is related with a convergence zone. The second one is located near the exit of the valley and is related with a divergence zone. The first region of $u=0$ is the mathematical condition for the channeling when the outgoing flow is perpendicular to the valley. This region is also related with the stagnation point.

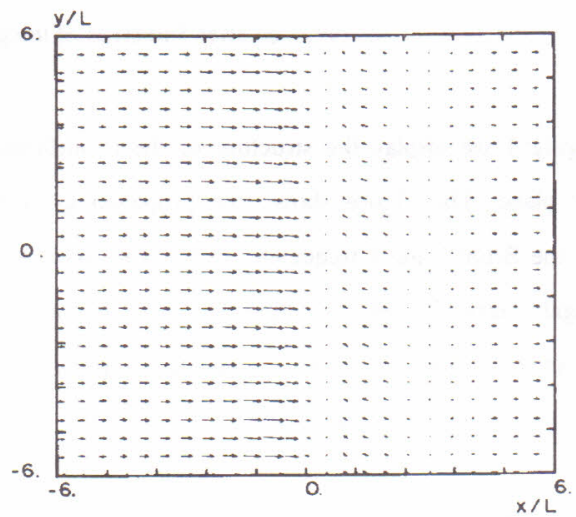

Figure 3. Plan view of velocity field at the lower surface for $F_{r}=0.33$ and $R_{o}=2.11$

In particular we can analyze the potential vorticity conservation. The numerical scheme employed (without the friction terms) does not yield a rigorous potential vorticity conservation. Indeed, preliminary integrations with the model showed that little changes of finite difference approximations to the potential vorticity may occur within the inviscid part of the fluid; these are just truncation erros, but we observe that the model and the scheme employed given a trends to the conservation. When the flow coming from the west and encounter a north-south valley the potential vorticity requires that the flow must acquire a cyclone curvature to the north. This is because $\Delta p$ becomes greater in absolute value as the air is stretch over the valley. As $\Delta p$ returns to 
the original value in the lee of the valley the flow will curve back toward the south. We can observe in figure 2 this behavior in the streamlines pattern.

Figure 3 shows a vector plot of the model flow field at the same level as in the figure 2. As can be seen, the flow near the surface moves down the valley sidewalls, converge in the center and flows out the valley.

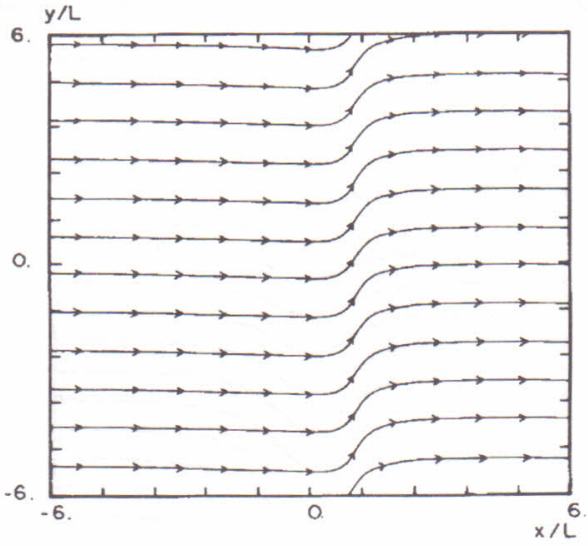

Figure 4. The same as Figure 2 for $F_{r}=1.33$ and $R_{o}=10.25$ The steady state was also obtained after 540 steps of integration.

Figure 4 illustrates the structure of the streamlines for $F_{r}=1.33$ and $R_{o}=10.52$. This experiment run for the same valley parameters and for the same flow speed but here we assume the Brunt-Vaisala frequency $N=0.01$ and the Coriolis parameter $f=2.53 \times 10^{-5} \mathrm{~s}^{-1}$ The steady state solution is obtained with the same time of integration, i.e. 540 time steps. In this experiment there is no formation of a low-level blocked region inside the valley, i.e. the $u$ velocity is greater than zero everywhere. As a consequence there are no observance of the onset of the secondary flow. When the Froude and Rossby numbers increases the role played by the buoyancy and the Coriolis force decrease. The increase of the Froude number is associated with the decrease of the Brunt-Vaisala frequency or the increase of the speed flow. In this situation there will be vertical motion and the fluid particles will deflected vertically following the terrain (this behavior is well know in a flow over a mountain). Particularly if the Froude number is greater than 1 the intrinsic frequency of the motions is greater than the buoyancy frequency and internal gravity waves are difficult to occur. We can observe this in figure 5 where we display the $\alpha$ surfaces, in a vertical cross section through the center plane, for the same experiment. We can compare figures 1 and 5 with the figure 1 of Smith (1979) who illustrates the steady inviscid flow over two-dimensional topography for a little or no 
influence of buoyancy (our figure 5) and for strong influence of buoyancy effects (our figure 1). On the other hand the increase of the Rossby number is associated with the increase of the speed flow, or the proximity of the Ecuador (decrease of latitude), or for valleys of small width. In all cases the time that a particle takes to cross the valley is small when compared with the characteristic time associated with the Coriolis force, and this force can be neglected. The importance of this term, in the momentum equations, for the along-valley wind is showed in figure 6 . Here we display an experiment which runs for the same parameters used in experiment showed in figures 1-3 but without the Coriolis terms. In this case the streamlines at lower surface is symmetric and there are no evidence of the channeling of the flow.

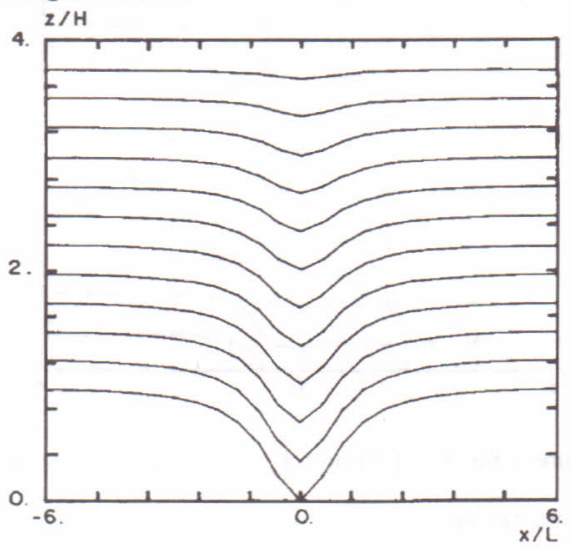

Figure 5. The same as Figure 1 for $F_{r}=1.33$ and $R_{o}=10.25$

The form nature of the solutions presented later enable us to search by the region in parameter space $\left(F_{r} \times R_{o}\right)$ where the channeling occur. In essence the flow enter the valley and the blocking occur for some critical value of $F_{r}$. But the channeling is not a result of this parameter only. Once the blocking is verified the Coriolis force begins to play its role in the resulting flow field. The effect of this force, associated with the influence of the Buoyancy force will determine the dynamics of air flows over a valley. The parameter space is then divided in two regions. For some part of this space the axial flow field will be observed and for the other part there will be no axial flow. We expected, for example, that for $R_{o} \rightarrow \infty$, i.e. flow without Coriolis force, the channeling will be not observed for any value of $F_{r}$. In the other extreme of the $R_{o}$ axis, i.e. $R_{o} \rightarrow \infty$ or flow with Coriolis term $\gg$ advection terms, the channeling will occur for $F_{r}$ lower than a critical value. To search this critical value as well the curve that divide the parameter space in two regions a great number of numerical experiments was done. When the result flow field was similar those described in figures 1-3 we get a point in the channeled region. When the result is similar those described by 
figures 4-5 we get a point in the no channeled region. To look the transition from one side to other side we proceed as follow. We fixed the Rossby number and allow the Froude number to sweep an interval between $0.1<F_{r}<2.0$. this procedure allows us to discover the transition point and as a consequence to draw the desired curve, which is presented in figure 7. The area below this curve is the channeled region.

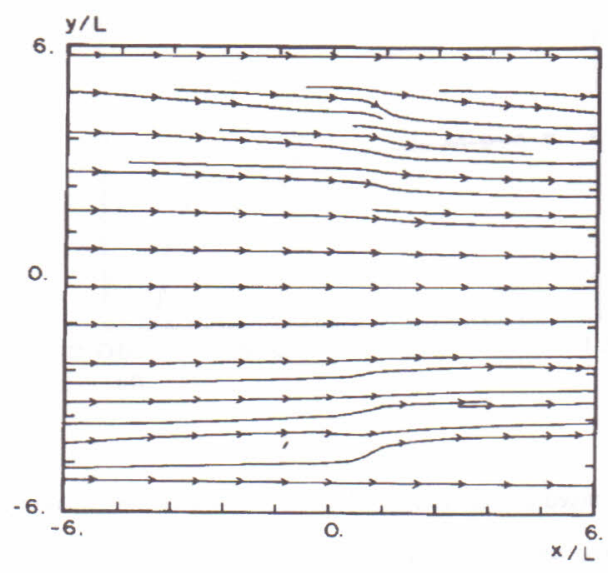

Figure 6. The steady state streamlines at the lower surface for $F_{r}=0.33$ and $R_{o}=2.11$ (the same values as Figure 2) but without the Coriolis terms in the momentum equations.

From this figure we observe that the critical Froude number for the channeling depends on the Rossby number. The maximum critical value is about 0.41 , i.e. for the maximum influence of the Coriolis force numerically simulated $\left(R_{o} \propto 0.2\right)$ the axial flow develops when $F_{r}<0.41$. For greater values of $R_{o}$ the critical Froude number decreases. When $R_{o} \propto 20.0$, for example, $F_{r_{c}} \propto 0.32$. Between these values of $R_{o}\left(0.2<R_{o}<20.0\right)$ the $F_{r_{c}}$ has a little variation $\left(0.41<F_{r}<0.32\right)$, but when $R_{o}$ is greater than 20.0 the $F_{r_{c}}$ goes quickly to zero.

There is one important observation to be made. The critical Froude number observed for the channeling is different from that observed for the stagnation. This second one is greater than the first one. Intuitively we expected to observe this fact. The tendency for the breaking and stagnation of the surface level flow comes before of the incoming flow acquires kinetic energy in the axial direction. Once the stagnation occurs there are two ways for the onset of the channeling, i.e. decreasing the Rossby number or decreasing the Froude number. 


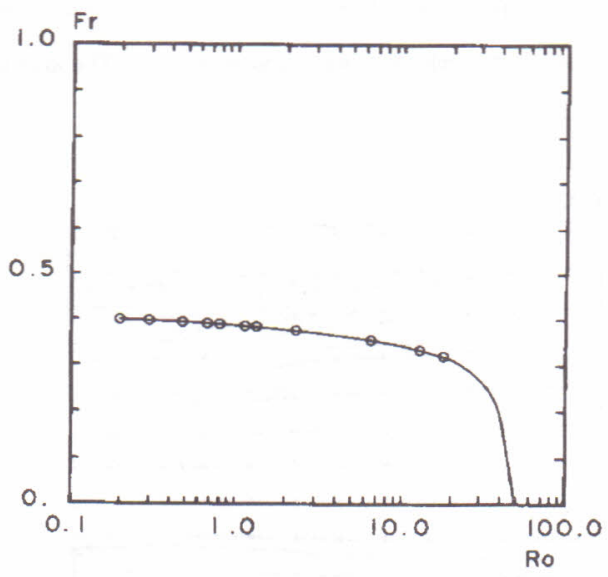

Figure 7. Sketch of the channeled and no-channeled regions in the space of parameters. In the region below the full line the wind parallel to the axis of the valley is observed

\section{CONCLUSIONS}

In this paper it was numerically simulated an air stream perpendicularly incident upon a valley in order to determine the influence of the Froude and the Rossby numbers in the pattern of the flow. Particularly attention was focused under what range of these parameters this air stream will be channeled in the valley. The results shows that the channeling occur if the values of these numbers are limited in one region in the space defined by them. For $R_{o} \rightarrow 0$, i.e. for the maximum influence of the Coriolis force, the channeling is observed for $F_{r} \leq 0.41$. When the $R_{o}$ increase the value of the $F_{r}$ decrease. In other words, when the Coriolis force becomes smaller more strong stable stratification is required for the channeling of the wind in a valley. However for $R_{o} \geq 50$ (weak influence of the Coriolis force) the incident air stream will not be channeled for any value of $F_{r}$. In this case the winds follows the topography.

The channeling here obtained are expected in very narrow and deep valleys as well in extremely broad and flat valleys, however as it was neglected the friction the results here obtained are expected only for broad valleys and dubious for narrow valleys. 


\section{REFERENCES}

P. K. Smolarkiewicz and R. Rotunno, 1988: Low Froude Number Past Three-Dimensional Obstacles. Part I: Baroclinically Generated Lee Vortices, J. Atmos. Sci., vol.46, 1154-1164.

F. Defant, 1951: Local Winds. In: "Compendium of Meteorology" (T. F. Malone, ed.), American Meteorological Society, Boston, 655-672.

J. Egger, 1981: Thermally Forced Circulations in a Valley, Geophys. Astrophys. Dyn., vol.17, 255279.

R. T. McNider and R. A. Pielke, 1984: Numerical Simulation of Slope and Mountain Flows, J. Clim. Appl. Meteorol., vol.23, 1441-1453.

G. Adrian and F. Fiedler, 1991: Simulation of Unstationary Wind and Temparature Fields over Complex Terrain and Comparision with Observations, Beitr. Phys. Atmosph., vol.64, 27-48.

F. Wippermann, 1984: Air Flow over and in a Broad Valleys: Channeling and Counter-Current, Beitr. Phys. Atmosph., vol.57, 92-105.

I. Vergeiner and E. Dreiseitl, 1987: Valley Winds and Slope Winds- Observations and Elementary Thoughts, Meteorol. Atmosph. Phys., vol.36, 264-286.

R. B. Smith, 1979: The Influence of Mountains on the Atmosphere, In: "Advances of Geophysics", vol.21, 87-230.

D. R. Fitzjarrald and G. G. Lala, 1989: Hudson Valley Fog Environments, J. Appl. Meteorol., vol.28, 1303-1328.

P. K. Smolarkiewicz, 1991: On Forward-in-Time Differencing for Fluids, Preprint, NCAR-Boulder, Co.

P. K. Smolarkiewicz and T. L. Clark, 1986: The Multidimensional Positive Definite Advection Transport Algorith: Further Development and Applications, J. Comput. Phys., vol.67, 396-438. 
